\title{
Technical Note: Trend estimation from irregularly sampled, correlated data
}

\author{
T. von Clarmann, G. Stiller, U. Grabowski, E. Eckert, and J. Orphal \\ Karlsruhe Institute of Technology, Institute for Meteorology and Climate Research, Karlsruhe, Germany \\ Received: 16 November 2009 - Published in Atmos. Chem. Phys. Discuss.: 21 December 2009 \\ Revised: 12 July 2010 - Accepted: 12 July 2010 - Published: 22 July 2010
}

\begin{abstract}
Estimation of a trend of an atmospheric state variable is usually performed by fitting a linear regression line to a set of data of this variable sampled at different times. Often these data are irregularly sampled in space and time and clustered in a sense that error correlations among data points cause a similar error of data points sampled at similar times. Since this can affect the estimated trend, we suggest to take the full error covariance matrix of the data into account. Superimposed periodic variations can be jointly fitted in a straightforward manner, even if the shape of the periodic function is not known. Global data sets, particularly satellite data, can form the basis to estimate the error correlations. State-dependent amplitudes of superimposed periodic corrections result in a non-linear optimization problem which is solved iteratively.
\end{abstract}

\section{Introduction}

Correct trend estimation is a key question in the discussion of climate change (IPCC, 2007). While fitting a straight line to a sample of data is an almost trivial task, errors in the data set and non-representativeness of the sample add some difficulty to the problem. Assuming normally distributed errors which are uncorrelated over the sample, each data point is simply weighted by the inverse of its variance to obtain a best linear unbiased estimate of the trend (Aitken, 1935). Methods applicable to least squares fitting of data where both the dependent and the independent variables are affected by errors have recently been reviewed by Cantrell (2008).

If the assumption of normal error distribution is questionable, robust linear regression methods help to reduce the sensitivity of the trend to outliers in the sample (Muhlbauer

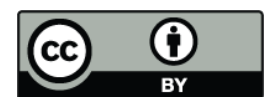

Correspondence to: $\mathrm{T}$. von Clarmann (thomas.clarmann@kit.edu) et al. 2009 and references therein). Another cure against nonnormality of distributions of residuals are bootstrap methods, introduced by Efron (1979) as a variant to jackknife methods and applied to atmospheric trend analysis by, e.g. Cox et al. (2002), Gardiner et al. (2008) or Vigouroux et al. (2008).

Besides non-normality of the distribution of residuals, correlations between the sampled data are another class of problems. When using multisite means to infer a trend, the standard errors of the means $\sigma_{\text {mean }}$ which determine the weight of each mean in the regression analysis are not the standard deviation $\sigma$ of the sample over the sites divided by the square root of the number of sites $M$ but

$\sigma_{\text {mean }}=\sqrt{\sigma^{2}\left(\frac{1+(M-1) \bar{r}_{\text {inter }}}{M}\right)}$,

where $\bar{r}_{\text {inter }}$ is the average intersite correlation coefficient (Jones et al., 1997). This can easily be verified by multiplication of the averaging operator from the left and right to the intersite covariance matrix $\mathbf{S}_{\text {inter }}$ according to multivariate Gaussian error propagation:

$\sigma_{\text {mean, inter }}^{2}=\left(\frac{1}{M}, \ldots, \frac{1}{M}\right) \mathbf{S}_{\text {inter }}\left(\begin{array}{c}\frac{1}{M} \\ \vdots \\ \frac{1}{M}\end{array}\right)$,

where the element at position $(i, k)$ of $\mathbf{S}_{\text {inter }}$ is $r_{\text {inter } i, k} \sigma^{2}$. This approach solves the problem of intersite correlations and is applicable, e.g., if measurements of the same set of sites are used over the whole period. $\sigma_{\text {mean }}$ calculated under consideration of $\bar{r}_{\text {inter }}$ accounts for the fact that the available sites do not fully represent the population, i.e., the sample mean at a given time is not necessarily identical to the global mean. Since the same set of stations is used over the whole period, the measurements at the given sites are not a random sample.

Weatherhead et al. (1998) discuss how autocorrelations of noise in the data affect the precision of the estimated trend,

Published by Copernicus Publications on behalf of the European Geosciences Union. 
and they provide a practical method to consider these autocorrelations to avoid over-optimistic confidence estimated with respect to inferred linear trends. Further, these authors present a tool to estimate the required length of the time series to significantly detect a trend.

None of these papers, however, tackles the problem of how to derive trends on the basis of inhomogeneous data sets. In this technical note, we investigate the problem that the sampled data are clustered in a sense that the data are groupwise correlated in the time domain. This is the case when data inhomogeneities cause systematic deviations between subsets of the data of time series. Through irregular sampling in time, these systematic deviations map onto the time series as errors correlated in the time domain. A typical example would be the estimation of a trend of one atmospheric state variable from measurements at two different latitudes, where one measurement site dominates the earlier part and the other measurement site the later part of the time series. The neglected latitudinal dependence of the observed quantity maps onto the time domain if the atmosphere is irregularly sampled at the different observation sites. Such data sets, where the target variable depends on further variables (e.g. latitude, calibration standard) except the independent variable of the regression analysis (e.g., time), we call inhomogeneous, and the unconsidered independent variables we call "hidden variables". Irregular sampling of inhomogeneous data leads to clustering, because certain values of the independent variable may go along with certain values of the hidden variable. This dependence can be formulated as correlations, typically the larger, the more similar the value of the hidden variable is. Other reasons for such kind of groupwise correlations, besides latitude-dependence, are: data based on multiple measurement systems relying on different calibration standards (Engel et al., 2009), combination of data from two measurement systems which cover different episodes like $\mathrm{H}_{2} \mathrm{O}$ measurements from HALOE (Randel et al., 2004; Rosenlof and Reid, 2008) and MIPAS (Milz et al., 2005); and data sets where the operation mode has been changed during the time interval under assessment e.g. MIPAS $\mathrm{H}_{2} \mathrm{O}$ measurements before (Milz et al., 2009) and after 2004 (von Clarmann et al., 2009), when the instrument was operated at different spectral resolutions.

All these systematic differences between subsets of the data causing data inhomogeneity can be described as error correlations, which, if neglected, will not only render the significance analysis of the trend insignificant, but can actually change the slope of the regression line, i.e. lead to different trends.

In Sect. 2 of this paper we present a closed-form solution to infer a linear regression line from correlated measurements. In Sect. 3 we discuss the issue of seasonal or other periodic corrections and propose formalisms to infer these corrections directly from the measurements. Applicability of each of the schemes proposed will be demonstrated on the basis of selected case studies. While the proposed concept is quite straightforward rather than novel, we hope that it may be useful to the climate research community where currently error covariances in irregularly sampled data often seem to be ignored, even when inhomogeneous datasets are analyzed.

\section{Linear trends of clustered data}

Assuming a linear trend, we can approximate the temporal development of an atmospheric state variable $y$ as a straight line. A straight line is defined as

$\hat{y}(x ; a, b)=a+b x$,

where the symbol indicates a modeled or estimated rather than a measured state variable. In our application $x$ is the time of the measurement, but this concept of regression of clustered data is applicable to a wider context.

For normally distributed, but possibly interdependent errors of $y_{n}, n=1 \ldots N, N \geq 2$, of which the ex ante ${ }^{1}$ estimates are represented by the $N \times N$ covariance matrix $\mathbf{S}_{y}$, this straight line is the optimal regression line for which the cost function

$\chi^{2}=(\boldsymbol{y}-(a \boldsymbol{e}+b \boldsymbol{x}))^{T} \mathbf{S}_{y}{ }^{-1}(\boldsymbol{y}-(a \boldsymbol{e}+b \boldsymbol{x}))$

is minimum, where $\boldsymbol{e}=(1, \ldots, 1)^{T}$ and $\boldsymbol{x}=\left(x_{1}, \ldots, x_{N}\right)^{T}$, $\boldsymbol{y}=\left(y_{1}, \ldots, y_{N}\right)^{T}$, and ${ }^{T}$ denotes the transpose of a matrix. Coefficients $a$ and $b$ are inferred in a well established manner by setting the derivatives $\partial \chi^{2} / \partial a$ and $\partial \chi / \partial b$ to zero. This gives

$$
\begin{aligned}
& \frac{\partial \chi^{2}}{\partial a}=-2 \boldsymbol{e}^{T} \mathbf{S}_{y}^{-1}(\boldsymbol{y}-a \boldsymbol{e}-b \boldsymbol{x})=0 \\
& \boldsymbol{e}^{T} \mathbf{S}_{y}^{-1} \boldsymbol{y}=\boldsymbol{e}^{T} \mathbf{S}_{y}^{-1} a \boldsymbol{e}+\boldsymbol{e}^{T} \mathbf{S}_{y}^{-1} b \boldsymbol{x} \\
& a=\frac{\boldsymbol{e}^{T} \mathbf{S}_{y}^{-1} \boldsymbol{y}-\boldsymbol{e}^{T} \mathbf{S}_{y}^{-1} b \boldsymbol{x}}{\boldsymbol{e}^{T} \mathbf{S}_{y}^{-1} \boldsymbol{e}}
\end{aligned}
$$

and

$$
\begin{gathered}
\frac{\partial \chi^{2}}{\partial b}=-2 \boldsymbol{x}^{T} \mathbf{S}_{y}^{-1}(\boldsymbol{y}-a \boldsymbol{e}-b \boldsymbol{x})=0 \\
\boldsymbol{x}^{T} \mathbf{S}_{y}^{-1} \boldsymbol{y}-\boldsymbol{x}^{T} \mathbf{S}_{y}^{-1} a \boldsymbol{e}-\boldsymbol{x}^{T} \mathbf{S}_{y}^{-1} b \boldsymbol{x}=0 \\
\boldsymbol{x}^{T} \mathbf{S}_{y}^{-1} \boldsymbol{y}=\boldsymbol{x}^{T} \mathbf{S}_{y}^{-1} b \boldsymbol{x}+\boldsymbol{x}^{T} \mathbf{S}_{y}^{-1} a \boldsymbol{e} .
\end{gathered}
$$

Combining Eqs. 5 and 6 gives

$$
\begin{aligned}
& \boldsymbol{x}^{T} \mathbf{S}_{y}^{-1} \boldsymbol{y}=\boldsymbol{x}^{T} \mathbf{S}_{y}^{-1} b \boldsymbol{x}+\boldsymbol{x}^{T} \mathbf{S}_{y}^{-1} a \boldsymbol{e} \\
& =\boldsymbol{x}^{T} \mathbf{S}_{y}^{-1} b \boldsymbol{x}+\boldsymbol{x}^{T} \mathbf{S}_{y}^{-1} \boldsymbol{e} \frac{\boldsymbol{e}^{T} \mathbf{S}_{y}^{-1} \boldsymbol{y}-\boldsymbol{e}^{T} \mathbf{S}_{y}^{-1} b \boldsymbol{x}}{\boldsymbol{e}^{T} \mathbf{S}_{y}^{-1} \boldsymbol{e}} .
\end{aligned}
$$

\footnotetext{
${ }^{1}$ Ex ante error estimates we call error estimates based on propagation of assumed primary errors through the system, which can be calculated before the measurement actually has been made, as opposed to ex post error estimates which are based on the standard deviation of a sample of measurements (von Clarmann, 2006).
} 
This can be rearranged as

$\boldsymbol{x}^{T} \mathbf{S}_{y}{ }^{-1} b \boldsymbol{x}-\frac{\boldsymbol{x}^{T} \mathbf{S}_{y}^{-1} \boldsymbol{e} \boldsymbol{e}^{T} \mathbf{S}_{y}^{-1} b \boldsymbol{x}}{\boldsymbol{e}^{T} \mathbf{S}_{y}^{-1} \boldsymbol{e}}=$

$\boldsymbol{x}^{T} \mathbf{S}_{y}{ }^{-1} \boldsymbol{y}-\frac{\boldsymbol{x}^{T} \mathbf{S}_{y}^{-1} \boldsymbol{e} e^{T} \mathbf{S}_{y}^{-1} \boldsymbol{y}}{\boldsymbol{e}^{T} \mathbf{S}_{y}^{-1} \boldsymbol{e}}$

and finally solved to give $b$ :

$b=\frac{\boldsymbol{x}^{T} \mathbf{S}_{y}^{-1} \boldsymbol{y}-\frac{\boldsymbol{x}^{T} \mathbf{S}_{y}^{-1} e e^{T} \mathbf{S}_{y}^{-1} \boldsymbol{y}}{\boldsymbol{e}^{T} \mathbf{S}_{y}^{-1} \boldsymbol{e}}}{\boldsymbol{x}^{T} \mathbf{S}_{y}^{-1} \boldsymbol{x}-\frac{\boldsymbol{x}^{T} \mathbf{S}_{y}^{-1} e \boldsymbol{e}^{T} \mathbf{S}_{y}^{-1} \boldsymbol{x}}{\boldsymbol{e}^{T} \mathbf{S}_{y}^{-1} \boldsymbol{e}}}$

$=\frac{\boldsymbol{x}^{T} \mathbf{S}_{y}^{-1} \boldsymbol{y} \boldsymbol{e}^{T} \mathbf{S}_{y}^{-1} \boldsymbol{e}-\boldsymbol{x}^{T} \mathbf{S}_{y}^{-1} e \boldsymbol{e}^{T} \mathbf{S}_{y}^{-1} \boldsymbol{y}}{\boldsymbol{x}^{T} \mathbf{S}_{y}^{-1} \boldsymbol{x} \boldsymbol{e}^{T} \mathbf{S}_{y}^{-1} e-\boldsymbol{x}^{T} \mathbf{S}_{y}^{-1} e \boldsymbol{e}^{T} \mathbf{S}_{y}^{-1} \boldsymbol{x}}$.

Inserting this into Eq. 5 allows to calculate $a$ :

$a=\frac{\boldsymbol{x}^{T} \mathbf{S}_{y}^{-1} \boldsymbol{y}-\boldsymbol{e}^{T} \mathbf{S}_{y}^{-1} \boldsymbol{x} b}{\boldsymbol{e}^{T} \mathbf{S}_{y}^{-1} \boldsymbol{e}}$

$=\frac{e^{T} \mathbf{S}_{y}^{-1} \boldsymbol{y}-\boldsymbol{e}^{T} \mathbf{S}_{y}^{-1} \boldsymbol{x} \frac{\boldsymbol{x}^{T} \mathbf{S}_{y}^{-1} y e^{T} \mathbf{S}_{y}^{-1} e-x^{T} \mathbf{S}_{y}^{-1} e e^{T} \mathbf{S}_{y}^{-1} y}{x^{T} \mathbf{S}_{y}^{-1} x e^{T} \mathbf{S}_{y}^{-1} e-x^{T} \mathbf{S}_{y}^{-1} e^{T} \mathbf{S}_{y}^{-1} x}}{e^{T} \mathbf{S}_{y}^{-1} e}$

$=\frac{\boldsymbol{e}^{T} \mathbf{S}_{y}^{-1} \boldsymbol{y} \boldsymbol{x}^{T} \mathbf{S}_{y}^{-1} \boldsymbol{x}-\boldsymbol{e}^{T} \mathbf{S}_{y}^{-1} \boldsymbol{x} \boldsymbol{x}^{T} \mathbf{S}_{y}^{-1} \boldsymbol{y}}{\boldsymbol{e}^{T} \mathbf{S}_{y}^{-1} \boldsymbol{e} \boldsymbol{x}^{T} \mathbf{S}_{y}^{-1} \boldsymbol{x}-\boldsymbol{e}^{T} \mathbf{S}_{y}^{-1} \boldsymbol{x} \boldsymbol{x}^{T} \mathbf{S}_{y}^{-1} \boldsymbol{e}}$.

For unity $\mathbf{S}_{y}$ this reduces to the widely used parameters $\tilde{a}$ and $\tilde{b}$ of a regression line for data points of uncorrelated errors of equal variance:

$\tilde{a}=\frac{\sum y_{n}}{N}-\tilde{b} \frac{\sum x_{n}}{N}$,

where

$\tilde{b}=\frac{N \sum x_{n} y_{n}-\sum x_{n} \sum y_{n}}{N \sum x_{n}^{2}-\left(\sum x_{n}\right)^{2}}$

The uncertainty of the slope $b$ is:

$\sigma_{b}^{2}=\left(\frac{\partial b}{\partial \boldsymbol{y}}\right) \mathbf{S}_{y}\left(\frac{\partial b}{\partial \boldsymbol{y}}\right)^{T}$

$=\left(\frac{e^{T} \mathbf{S}_{y}^{-1} e x^{T} \mathbf{S}_{y}^{-1}-x^{T} \mathbf{S}_{y}^{-1} e e^{T} \mathbf{S}_{y}^{-1}}{\boldsymbol{x}^{T} \mathbf{S}_{y}^{-1} x \boldsymbol{e}^{T} \mathbf{S}_{y}^{-1} e-x^{T} \mathbf{S}_{y}^{-1} e e^{T} \mathbf{S}_{y}^{-1} \boldsymbol{x}}\right)$.

$\mathbf{S}_{y}\left(\frac{e^{T} \mathbf{S}_{y}^{-1} e x^{T} \mathbf{S}_{y}^{-1}-x^{T} \mathbf{S}_{y}^{-1} e e^{T} \mathbf{S}_{y}^{-1}}{\boldsymbol{x}^{T} \mathbf{S}_{y}^{-1} x e^{T} \mathbf{S}_{y}^{-1} e-x^{T} \mathbf{S}_{y}^{-1} e e^{T} \mathbf{S}_{y}^{-1} x}\right)^{T}$,

where $\left(\frac{\partial b}{\partial y}\right)=\left(\frac{\partial b}{\partial y_{1}} \ldots \frac{\partial b}{\partial y_{N}}\right)$. The uncertainty of axis intercept $a$ is estimated accordingly:

$\sigma_{a}^{2}=\left(\frac{\partial a}{\partial \boldsymbol{y}}\right) \mathbf{S}_{y}\left(\frac{\partial a}{\partial \boldsymbol{y}}\right)^{T}$

$=\left(\frac{\boldsymbol{x}^{T} \mathbf{S}_{y}^{-1} \boldsymbol{x} \boldsymbol{e}^{T} \mathbf{S}_{y}^{-1}-\boldsymbol{e}^{T} \mathbf{S}_{y}^{-1} \boldsymbol{x} \boldsymbol{x}^{T} \mathbf{S}_{y}^{-1}}{\boldsymbol{e}^{T} \mathbf{S}_{y}^{-1} e \boldsymbol{x}^{T} \mathbf{S}_{y}^{-1} \boldsymbol{x}-\boldsymbol{e}^{T} \mathbf{S}_{y}^{-1} \boldsymbol{x} \boldsymbol{x}^{T} \mathbf{S}_{y}^{-1} \boldsymbol{e}}\right)$.

$\mathbf{S}_{y}\left(\frac{x^{T} \mathbf{S}_{y}^{-1} x e^{T} \mathbf{S}_{y}^{-1}-e^{T} \mathbf{S}_{y}^{-1} x x^{T} \mathbf{S}_{y}^{-1}}{\boldsymbol{e}^{T} \mathbf{S}_{y}^{-1} e x^{T} \mathbf{S}_{y}^{-1} x-e^{T} \mathbf{S}_{y}^{-1} x x^{T} \mathbf{S}_{y}^{-1} e}\right)^{T}$
From comparison of Eqs. (9) and (12) we see that the error correlations do not only change the estimated error of the trend but also affect the trend itself, e.g. rotate the regression line.

Evaluation of Eq. (9) requires knowledge of the covariance matrix $\mathbf{S}_{y}$. For some error sources such error assumptions are available and reasonable assumptions on correlations within a class of measurements can be made; if, e.g., different subsets of the data are based on different calibration standards, perfect correlation, i.e., $r=1$, is appropriate for the calibration error component within each such subset. The bias between the subsets has to be estimated, and a fully correlated block of which each element is the square of the estimated bias between the $n$-th and the first data subset has to be added to that part of the covariance matrix which represents the $n$-th data subset. The following equation shows the construction of a covariance matrix for a dataset composed of two data subsets biased against each other by an unknown offset whose absolute value is estimated at bias 2,1 :

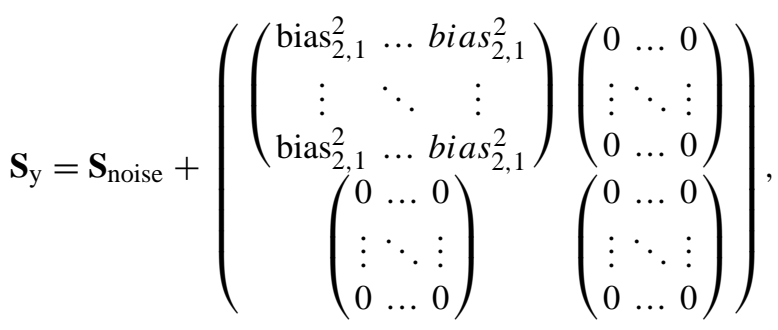

where $\mathbf{S}_{\text {noise }}$ is the measurement noise covariance matrix. For evaluation of error covariances representing other error sources, external data may be needed. Typical error correlations in a time series can be caused by the fact that the sample is composed of measurements at various locations. If the mean measurement times at two locations differ, any difference in the expectation value of the state variable with, e.g., latitude, will map onto the trend. If the latitudinal dependence is too complicated for a simple correction, or if there is a non-negligible residual location-related error even after correction, the related error correlation should be included in the covariance matrix $\mathbf{S}_{y}$. Covariances between sites $i$ and $k$ can be estimated from $N$ global satellite data sets as

$r_{i, k} \sigma_{i} \sigma_{k}=\sum_{n=1}^{N} \frac{\left(x_{i, n}-\bar{x}_{i}\right)\left(x_{k, n}-\bar{x}_{k}\right)}{N-1}$,

where $r_{i, k}$ is the correlation coefficient between sites $i$ and $k$. Three caveats have to be noted in this context:

(a) the $N$ global data sets should be measured in a time window short enough to justify neglect of any trend;

(b) when Eq. (16) is used to derive the variance (i.e. $i=k$ ) characterizing the representativeness of site $i$, the variance representing the satellite data measurement error must be subtracted; if the measurement errors of the satellite data are intercorrelated, the respective covariance matrix has to be subtracted from the covariance 
Table 1. Case Study 1.

\begin{tabular}{rrllll}
\hline Case & $\Delta T^{(1)}$ & $\begin{array}{l}\sigma_{\Delta T}^{2(2)} \\
(\mathrm{K})\end{array}$ & $r^{(3)}$ & $\begin{array}{l}a \\
(\mathrm{~K})\end{array}$ & $\begin{array}{l}b \\
(\mathrm{~K} / \mathrm{yr})\end{array}$ \\
\hline 1.1 & 0.0 & 0.31 & 1 & $217.77 \pm 0.80$ & $0.05 \pm 0.10$ \\
1.2 & 0.0 & 0.0 & 0 & $217.84 \pm 0.57$ & $0.04 \pm 0.08$ \\
1.3 & 0.0 & 0.31 & 0 & $217.88 \pm 0.66$ & $0.03 \pm 0.08$ \\
1.4 & -2.0 & 4.0 & 1 & $216.72 \pm 1.91$ & $0.18 \pm 0.27$ \\
1.5 & -2.0 & 0.0 & 0 & $215.00 \pm 0.57$ & $0.41 \pm 0.08$ \\
1.6 & -2.0 & 4.0 & 0 & $216.24 \pm 0.87$ & $0.25 \pm 0.11$ \\
\hline
\end{tabular}

(1) $\Delta T$ : artificial bias added to first three measurements;

(2) $\sigma_{\Delta T}^{2}$ : additional variance in the related block of $\mathbf{S}_{y}$ to account for bias;

(3) $r$ : correlation coefficient applied to related block of $\mathbf{S}_{y}$ to account for bias.

matrix derived according to Eq. (16) to obtain the intersite covariance matrix;

(c) the finite spatial resolution of satellite measurements might be an issue.

In any case, the covariance matrix describing the uncertainty due to the hidden variable is added to the - often diagonal - covariance matrix characterizing the measurement noise of the given sample. In a more general context where various error sources independent of each other are considered, the covariance matrix representing the total uncertainty of the dataset is calculated as the sum of the respective covariance matrices.

\section{Case Study 1:}

The trend estimator of Eq. 9 is applied to tropical $\left(30^{\circ} \mathrm{S}\right.$ to $30^{\circ} \mathrm{N}$ ) annual temperature averages at $25 \mathrm{~km}$ altitude inferred from limb infrared measurements recorded with the MIPAS instrument (Fischer et al., 2008). Temperature retrievals for the years 2002 to 2004 are based on measurements when MIPAS was operated at full spectral resolution (von Clarmann et al., 2003), while measurements from 2005 to 2009 are based on reduced spectral resolution measurements (von Clarmann et al., 2009). This different operation mode potentially causes an unknown bias between the subsets of data, which we estimate at $\pm 0.56 \mathrm{~K}$ and account for by adding

$$
\begin{aligned}
& \left(\begin{array}{l}
0.56 \mathrm{~K} \\
0.56 \mathrm{~K} \\
0.56 \mathrm{~K}
\end{array}\right)(0.56 \mathrm{~K} ; 0.56 \mathrm{~K} ; 0.56 \mathrm{~K})= \\
& \left(\begin{array}{l}
0.31 \mathrm{~K}^{2} ; 0.31 \mathrm{~K}^{2} ; 0.31 \mathrm{~K}^{2} \\
0.31 \mathrm{~K}^{2} ; 0.31 \mathrm{~K}^{2} ; 0.31 \mathrm{~K}^{2} \\
0.31 \mathrm{~K}^{2} ; 0.31 \mathrm{~K}^{2} ; 0.31 \mathrm{~K}^{2}
\end{array}\right)
\end{aligned}
$$

to the block of $\mathbf{S}_{y}$ which refers to the years 2002 to 2004 . The other error component is the estimated standard error of the

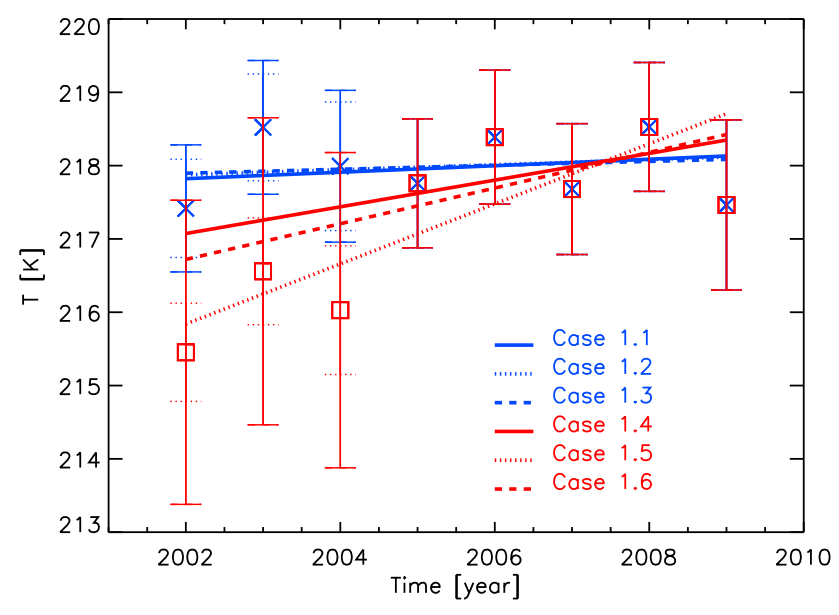

Fig. 1. Linear trends from MIPAS annual mean temperatures at $25 \mathrm{~km}$ altitude, $30^{\circ} \mathrm{S}-30^{\circ} \mathrm{N}$. Cases $1.1-1.3$ are based on measurement data as they are, while an artificial bias of $-2 \mathrm{~K}$ has been applied to temperatures of the years 2002-2004 in cases 1.4-1.6. In cases 1.1 and 1.4 covariances were treated correctly; in cases 1.2 and 1.5 the bias was neglected and in cases 1.3 and 1.6 only the variances of the bias were considered while its covariances were neglected. After 2004, data used for case studies 1.1-1.3 are identical to those used for case studies 1.4-1.6. Solid error bars are total errors while dotted error bars are random errors only.

annual mean, which is variable due to the different sample size for each year. The $x$-coordinate of our data set is the time since 2000, i.e. the intercept refers to the year 2000.

We calculate the regression parameters using the full covariance matrix (case 1.1) as described above, for a simplified case where the bias is ignored (case 1.2), and for a test case where only the variances of the bias are considered but not the covariances (case 1.3). Results are shown in Fig. 1 and compiled in Table 1.

Results seem to be quite robust with respect to the change of the MIPAS operation mode, i.e. the actual bias between the data subsets might be smaller than anticipated. Thus the correct statistical treatment of the bias is not critical. In none of the case studies there is a significant trend detected (we call a trend significant if it exceeds twice its uncertainty; for Gaussian errors this corresponds roughly to 5\% level of significance). In order to assess the robustness of the trend analysis scheme to data with large, correlated error components, we repeat the case studies mentioned above, but introduce an artificial bias of $-2 \mathrm{~K}$ to the data subset covering the years 2002 to 2004 and modify the covariance matrix accordingly (case 1.4). Larger data errors propagate to larger estimated errors in the regression parameters, but the scheme is robust in a sense that still no significant trend is produced. In case study 1.5 the same manipulated data are used, but the artificial error is neglected in the covariance matrix. The bias maps onto the regression parameters and causes an artificial, apparently significant trend. In case study 1.6 , the 
data uncertainty is considered as an uncorrelated error, i.e., the additional variances are considered but covariances are set to zero. The artificial trend still appears to be significant. This proves the importance of correct consideration of covariances. This example has been chosen for demonstration purposes. We neither claim that all possible error correlations in the MIPAS data have been perfectly quantified, nor should the inferred trends be used for extrapolation towards longer term analysis.

\section{Consideration of the annual cycle and related problems}

A linear trend may be superimposed with a periodic function of known periodic time, e.g. diurnal or seasonal variation, etc. There are several options to tackle this problem.

If the sample is large enough, the linear trend can be evaluated for subsets of data recorded at the same phase of the periodical variation, and the overall trend is calculated as an optimal (i.e. weighted inversely by the variances) mean of the individual trends. This requires binning of data; in the case of a seasonal cycle, the linear trend can be estimated as the mean of the trend over all Januaries, Februaries, etc. Problems occur when the amplitude of the seasonal cycle has a trend in itself and the whole observation time interval is not an integer multiple of the time of one cycle.

Another option is subtraction of the periodic signal prior to trend estimation. The periodic component of the signal can either be imported from an external source (model, independent data) or from the sample itself. The latter approach is not quite trivial, because the inferred mean periodical signal will, in turn, depend on the trend (periodic analysis usually is defined only for stationary time series, i.e. zero trend), such that either an iterative approach or a multivariate optimization (see below) is required. Care has to be taken to consider the reduction of degrees of freedom implied by inferring the correction from the data themselves. In the following, we will discuss how the periodic correction can be estimated simultaneously with the trend.

\subsection{Correction by sine and cosine functions}

The problem of the non-stationary nature of time series, which is by definition inherent in trend analysis, can be solved by retrieving the trend, the amplitude of the periodic variation, and possibly the phase and the shape of the oscillation in one step. In the case of a known function of unknown amplitude (e.g. sine), the amplitude can be fitted along with the trend. In the case of unknown phase, it is usually more appropriate to fit amplitudes of a sine and a cosine of the same period length rather than the amplitude and the phase, in order to keep the fit linear. A regression model involving a linear trend superimposed with a single harmonic variation of unknown phase but known period length $l$ is written as

$\hat{y}(x ; a, b, c, d)=a+b x+c \sin \frac{2 \pi x}{l}+d \cos \frac{2 \pi x}{l}$.

Setting the partial derivatives of

$\chi^{2}=(\boldsymbol{y}(\boldsymbol{x})-\hat{\boldsymbol{y}}(\boldsymbol{x}))^{T} \mathbf{S}_{y}^{-1}(\boldsymbol{y}(\boldsymbol{x})-\hat{\boldsymbol{y}}(\boldsymbol{x}))$

with respect to the parameters of the regression model to zero gives

$$
\begin{aligned}
& \frac{\partial \chi^{2}}{\partial a}=-2 \boldsymbol{e}^{T} \mathbf{S}_{y}^{-1}\left(\boldsymbol{y}(\boldsymbol{x})-a \boldsymbol{e}-b \boldsymbol{x}-c \boldsymbol{v}_{\sin }-d \boldsymbol{v}_{\cos }\right)=0 \\
& \frac{\partial \chi^{2}}{\partial b}= \\
& -2 \boldsymbol{x}^{T} \mathbf{S}_{y}^{-1}\left(\boldsymbol{y}(\boldsymbol{x})-a \boldsymbol{e}-b \boldsymbol{x}-c \boldsymbol{v}_{\sin }-d \boldsymbol{v}_{\cos }\right)=0
\end{aligned}
$$

$\frac{\partial \chi^{2}}{\partial c}=$

$-2 \boldsymbol{v}_{\sin }^{T} \mathbf{S}_{y}^{-1}\left(\boldsymbol{y}(\boldsymbol{x})-a \boldsymbol{e}-b \boldsymbol{x}-c \boldsymbol{v}_{\sin }-d \boldsymbol{v}_{\cos }\right)=0$

$\frac{\partial \chi^{2}}{\partial d}=$

$-2 \boldsymbol{v}_{\cos }^{T} \mathbf{S}_{y}^{-1}\left(\boldsymbol{y}(\boldsymbol{x})-a \boldsymbol{e}-b \boldsymbol{x}-c \boldsymbol{v}_{\sin }-d \boldsymbol{v}_{\cos }\right)=0$,

where $\boldsymbol{v}_{\sin }=\left(\sin \frac{2 \pi x_{1}}{l}, \ldots, \sin \frac{2 \pi x_{N}}{l}\right)^{T}$, and

$\boldsymbol{v}_{\cos }=\left(\cos \frac{2 \pi x_{1}}{l}, \ldots, \cos \frac{2 \pi x_{N}}{l}\right)^{T}$. Equations (20-23) form a system of four equations linear in $a, b, c$ and $d$, of the type

$\mathbf{T}\left(\begin{array}{l}a \\ b \\ c \\ d\end{array}\right)=\boldsymbol{q}$

where

$$
\begin{aligned}
T_{1,1} & =2 \boldsymbol{e}^{T} \mathbf{S}_{y}^{-1} \boldsymbol{e} \\
T_{1,2} & =2 \boldsymbol{e}^{T} \mathbf{S}_{y}^{-1} \boldsymbol{x} \\
& \vdots \\
T_{4,4} & =2 \boldsymbol{v}_{\cos }^{T} \mathbf{S}_{y}^{-1} \boldsymbol{v}_{\cos } \\
q_{1} & =2 \boldsymbol{e}^{T} \mathbf{S}_{y}^{-1} \boldsymbol{y}(\boldsymbol{x}) \\
& \vdots \\
q_{4} & =2 \boldsymbol{v}_{\cos }^{T} \mathbf{S}_{y}^{-1} \boldsymbol{y}(\boldsymbol{x}) .
\end{aligned}
$$

For $N \geq 4$ and non-singularity of $\mathbf{T}$, Eq. (24) can be unambiguously solved for the four parameters $a \ldots d$. This can be done by any linear equation program package at hand. The advantage of this approach is that it does not require a stationary time series to evaluate the amplitudes of the oscillations. If need be, this type of analysis can also involve multiple periodic functions of different periods, which may be 
made subject to lowpass filtering. This generalization of the schemes presented here to applications with more than one pair of periodic functions is straightforward, and the relationship to harmonic analysis is obvious if the period lengths are chosen to be integer fractions of the longest one. Problems with singularity of $\mathbf{T}$ will occur if the number of data points is smaller than the number of period lengths plus 2 .

The covariance matrix $\mathbf{S}_{a, b, c, d}$ of the regression parameters is

$$
\begin{aligned}
& \mathbf{S}_{a, b, c, d}=\left(\begin{array}{c}
\frac{\partial a}{\partial y} \\
\frac{\partial b}{\partial y} \\
\frac{\partial c}{\partial y} \\
\frac{\partial d}{\partial \boldsymbol{y}}
\end{array}\right) \mathbf{S}_{y}\left(\begin{array}{c}
\frac{\partial a}{\partial y} \\
\frac{\partial b}{\partial \boldsymbol{y}} \\
\frac{\partial c}{\partial \boldsymbol{y}} \\
\frac{\partial d}{\partial \boldsymbol{y}}
\end{array}\right)^{T} \\
& =\left(\mathbf{T}^{-1}\left(\frac{\partial \boldsymbol{q}}{\partial \boldsymbol{y}}\right)\right) \mathbf{S}_{y}\left(\mathbf{T}^{-1}\left(\frac{\partial \boldsymbol{q}}{\partial \boldsymbol{y}}\right)\right)^{T} \\
& =\left(\mathbf{T}^{-1}\left(\begin{array}{c}
2 \boldsymbol{e}^{T} \mathbf{S}_{y}^{-1} \\
2 \boldsymbol{x}^{T} \mathbf{S}_{y}^{-1} \\
2 \boldsymbol{v}_{\sin }^{T} \mathbf{S}_{y}^{-1} \\
2 \boldsymbol{v}_{\cos }^{T} \mathbf{S}_{y}^{-1}
\end{array}\right)\right) \mathbf{S}_{y} \\
& \times\left(\mathbf{T}^{-1}\left(\begin{array}{c}
2 \boldsymbol{e}^{T} \mathbf{S}_{y}^{-1} \\
2 \boldsymbol{x}^{T} \mathbf{S}_{y}^{-1} \\
2 \boldsymbol{v}_{\sin }^{T} \mathbf{S}_{y}^{-1} \\
2 \boldsymbol{v}_{\cos }^{T} \mathbf{S}_{y}^{-1}
\end{array}\right)\right)^{T} .
\end{aligned}
$$

The off-diagonal elements of $\mathbf{S}_{y}$ will determine whether the data errors map either predominantly onto the slope or onto the axis intercept of the regression curve. For example, large positive correlations throughout the data lead to large intercept errors while the slope remains quite well determined with sometimes surprisingly small uncertainties. The extreme case would be fully correlated data errors. It is well known that such a constant bias in the data does not affect the trend at all. Consideration of full covariance matrices allows the correct treatment of realistic cases, where the errors are neither purely random nor purely systematic but may include correlations within subsets of the data.

The uncertainties estimated by Eq. (26) include the propagation of data errors onto the regression parameters but not uncertainties caused by the use of an inappropriate model (e.g. neglect of higher order or periodic components).

\subsubsection{Case Study 2:}

The trend estimator of Eqs. (18-25) is applied to tropical $\left(30^{\circ} \mathrm{S}\right.$ to $\left.30^{\circ} \mathrm{N}\right)$ "pseudomonthly" MIPAS temperature averages at $25 \mathrm{~km}$. A "pseudomonth" we call a time period of 32 days, chosen to be an integer division of the mean period of the semi-annual oscillation (SAO) which was first detected by Reed (1965). The period of the SAO in the MIPAS data set is estimated at 192 days, which is in good agreement with SAO period length of about 194 days at $30 \mathrm{~km}$ as reported by Guharay et al. (2009). We extend the method
Table 2. Case Study 2.

\begin{tabular}{lrlllr}
\hline Case & $\begin{array}{r}\Delta T^{(1)} \\
(\mathrm{K})\end{array}$ & $\begin{array}{l}\sigma_{\Delta T}^{2(2)} \\
\left(\mathrm{K}^{2}\right)\end{array}$ & $r^{(3)}$ & $\begin{array}{l}a \\
(\mathrm{~K})\end{array}$ & $\begin{array}{r}b \\
(\mathrm{~K} / \mathrm{yr})\end{array}$ \\
\hline 2.1 & 0.0 & 0.31 & 1 & $219.24 \pm 0.25$ & $-0.09 \pm 0.03$ \\
2.2 & 0.0 & 0.0 & 0 & $219.14 \pm 0.10$ & $-0.08 \pm 0.02$ \\
2.3 & 0.0 & 0.31 & 0 & $218.75 \pm 0.28$ & $-0.04 \pm 0.04$ \\
2.4 & -2.0 & 4.0 & 1 & $219.24 \pm 0.26$ & $-0.09 \pm 0.03$ \\
2.5 & -2.0 & 0.0 & 0 & $215.83 \pm 0.10$ & $0.35 \pm 0.02$ \\
2.6 & -2.0 & 4.0 & 0 & $215.70 \pm 0.91$ & $0.34 \pm 0.14$ \\
\hline
\end{tabular}

(1) $\Delta T$ : artificial bias added to first three measurements;

(2) $\sigma_{\Delta T}^{2}$ : additional variance in the related block of $\mathbf{S}_{y}$ to account for bias;

(3) $r$ : correlation coefficient applied to related block of $\mathbf{S}_{y}$ to account for bias.

described in the theory part towards two sine and two cosine terms as to include also the quasi-biennial oscillation (c.f. Baldwin et al. 2001). Its period length is assumed to be 25 months, in agreement with data provided by Freie Universität Berlin (http://www.geo.fu-berlin.de/met/ag/strat/ produkte/qbo/index.html). The case studies for this application, particularly the treatment of biases, were selected as in case study 1.

Correct treatment of the original MIPAS data and their correlations (case study 2.1) results in a negative trend, neglect of correlations (case study 2.2) and their simplified treatment (case study 2.3) lead to small negative temperature trends (Table 2 and Fig. 2). The trend obtained from case study 2.3 is insignificant. More interesting is the assessment of the data superimposed with an artificial bias: If the bias is considered correctly in the covariance matrix (case study 2.4), it does not change the trend by any substantial amount, while otherwise (case studies 2.5 and 2.6) there is an apparent positive trend, which appears significant. This demonstrates how powerful the concept of bias consideration in the error covariance matrix is to remedy data inhomogeneities.

\subsection{Correction by a discrete empirical function}

If the shape of the periodic variation is not known a priori, it can be inferred from the data themselves in one step with the trend estimation. For data binned in the time domain (e.g. when monthly means are used to infer a trend with superimposed seasonal variation) corrections for each phase (e.g. monthly corrections) are fitted along with slope and axis intercept. The regression model then is

$\hat{y}=a+b x+c_{j(x)}$,

where $c_{j(x)}$ is the (e.g., monthly) correction applicable to the measurement made at time $x$. The cost function to be minimized for this application is 


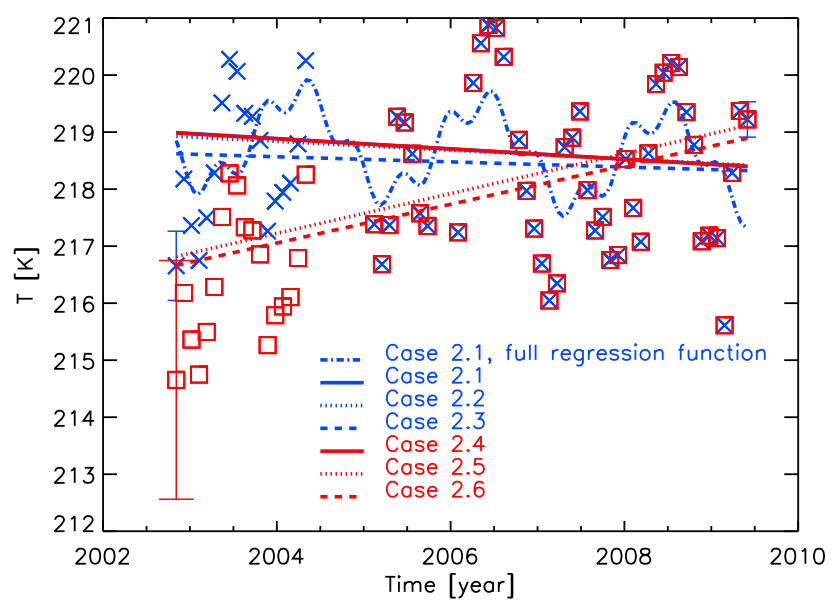

Fig. 2. Linear trends from MIPAS 32-day mean temperatures at $25 \mathrm{~km}$ altitude, $30^{\circ} \mathrm{S}-30^{\circ} \mathrm{N}$, with consideration of sine and cosine corrections for the quasi-biennial oscillation and the semi-annual oscillation. Test cases represent different treatment of the bias, for details see Fig. 1. The linear term of the regression function is shown for all cases 2.1-2.6, while, for reasons of clarity, the full regression curve is shown for case 2.1 only. Representative error bars include both the random error and - if considered in the respective case - the bias.

$\chi^{2}=$

$(\boldsymbol{y}(\boldsymbol{x})-(a \boldsymbol{e}+b \boldsymbol{x}+\mathbf{U} \boldsymbol{c}))^{T} \mathbf{S}_{y}^{-1}(\boldsymbol{y}(\boldsymbol{x})-(a \boldsymbol{e}+b \boldsymbol{x}+\mathbf{U} \boldsymbol{c}))$

where $c$ is a vector of length $J$, where $J$ is the number of phase bins ( $J=12$ in the case of monthly corrections in an annual cycle) representing the applicable corrections for each phase bin, and $\mathbf{U}$ is a selection matrix with all elements in the $n$-th row zero except for column $j$, where $j$ represents the month when measurement $x_{n}$ was made, where the matrix element is one. $N \geq J+2$ is required, and for each phase bin at least one measurement must be available. Minimization of $\chi^{2}$ to get the regression parameters follows the same strategy as described in Sect. 3, i.e. the derivatives of $\chi^{2}$ with respect to the regression parameters $a, b$ and $c$ are set zero and the resulting system of linear equations is solved. However, the derivative with respect to the axis intercept and that with respect to the additive corrections lead to linearly dependent equations. Thus, the equation

$\frac{\partial \chi^{2}}{\partial a}=0$

is dropped for reasons of redundancy, and

$\sum_{j=1}^{J} c_{j}=0$

is included to constrain the solution to zero mean periodic corrections.

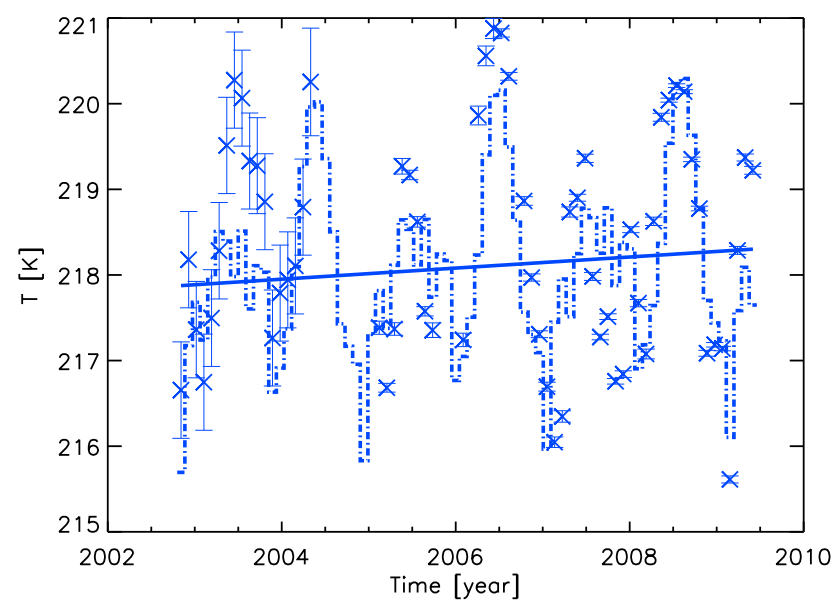

Fig. 3. Linear trends from MIPAS 32-day mean temperatures at $25 \mathrm{~km}$ altitude, $30^{\circ} \mathrm{S}-30^{\circ} \mathrm{N}$, using the discrete empirical periodical correction, as described in Sect. 3.2. Both the linear part of the regression function and the complete regression function are shown. No artificial bias has been added to the data in this case study.

\section{Case Study 3:}

Here we use the same binned MIPAS temperature data as for case studies 2.1-2.3. A periodicity of 25 bins is assumed to match the quasi-biennial oscillation. The trend is estimated at $0.06 \pm 0.01 \mathrm{~K} / \mathrm{yr}$ (Fig. 3). The estimated uncertainty is the propagation of the measurement error on the trend and includes the random error and the bias only; no other error sources are considered; particularly temperatures might not be perfectly described by the regression model chosen, and the time series of limited length might not be representative for a longer term. As a side aspect, differences in the trends compared to those inferred in case study 2 (Fig. 2, c.f. case 2.1) demonstrate the importance of the choice of the regression model.

\subsection{Correction by a continuous empirical function}

If binning or averaging in the time domain is to be avoided, the discrete application of the correction can be replaced by a continuous time-dependence. This might be advisable if data are available on a time-grid finer than the bin-width, and if the variation of $y$ within a bin is large. The actual correction for a given $y(x)$ can then be estimated by, e.g., linear, interpolation, leading to the regression model

$\hat{y}=a+b x+c_{j(x)} w_{n, j(x)}+c_{j(x)+1} w_{n, j(x)+1}$

where $c$ are periodic corrections at predefined phases and $v$ their weights. More specifically, $c_{j(x)}$ and $c_{j(x)+1}$ are the periodic corrections referring to the closest times before/after the measurement time $x$ (e.g. for each 1st of the month when 
the measurement was made, and the 1st of the following month, respectively). The weighting factors $w$ are

$w_{n, j(x)}=\frac{d_{j(x)}-d\left(x_{n}\right)}{d_{j(x)}}$

and

$w_{n, j(x)+1}=\frac{d\left(x_{n}\right)}{d_{j(x)}}$,

where $d_{j(x)}$ is the difference between the times related to $c_{j(x)}$ and $c_{j(x)+1}$ (e.g. the number of days of the month) and $d(x)$ is difference between the actual measurement time and the time related to $c_{j(x)}$ (e.g. the day of the month). Periodicity is assumed in a sense that $c_{j(x)}=c_{j\left(x+\sum d_{j}\right)}$. The number of parameters to be fitted still is $J+2$, as with the approach involving binning in the time domain. The cost function to be minimized for this application has the same structure as Eq. (28):

$\chi^{2}=$

$(\boldsymbol{y}(\boldsymbol{x})-(a \boldsymbol{e}+b \boldsymbol{x}+\mathbf{W} \boldsymbol{c}))^{T} \mathbf{S}_{y}^{-1}(\boldsymbol{y}(\boldsymbol{x})-(a \boldsymbol{e}+b \boldsymbol{x}+\mathbf{W} \boldsymbol{c}))$

$\mathbf{W}$ is a matrix with all elements in the $n$-th row zero except for column $j$ and $j+1$ (or 1 , if $j$ denotes the last column), where $j$ and $j+1$ represent the month when measurement $n$ was made, and the subsequent month, respectively. The respective matrix elements are the weights of the monthly correction factors $c_{j}$ and $c_{j+1}$, as defined by Eqs. (32-33). The minimization of the cost functions of Eqs. (28) and (34) and error estimation follows the same scheme as outlined for the cost function in Eq. (19), except that the zero mean constraint for $c_{1} \ldots c_{j}$ has to substitute the equation involving the partial derivative of $\chi^{2}$ with respect to $a$, as in Sect. 4.2.

\section{Case Study 4:}

In this case study we use MIPAS tropical $\left(30^{\circ} \mathrm{S}\right.$ to $\left.30^{\circ} \mathrm{N}\right)$ daily mean temperatures at $25 \mathrm{~km}$ altitude. As in case study 3, 27 independent periodic correction terms are jointly inferred from the data along with axis intercept and linear trend; contrary to case study 3 , the actual correction applicable to a data point is determined by linear interpolation between the two correction terms representing the nominal times before and after the actual measurement time. The inferred trend is $0.06 \pm 0.01 \mathrm{~K} / \mathrm{yr}$ (Fig. 4), as in case study 3. As in the preceding case studies, random errors and the bias due to the change in the MIPAS measurement mode are the only errors considered.

\subsection{Correction by functions of state-dependent amplitude}

Often the amplitude of the periodic variation depends linearly on the actual mean state $a+b x$ :

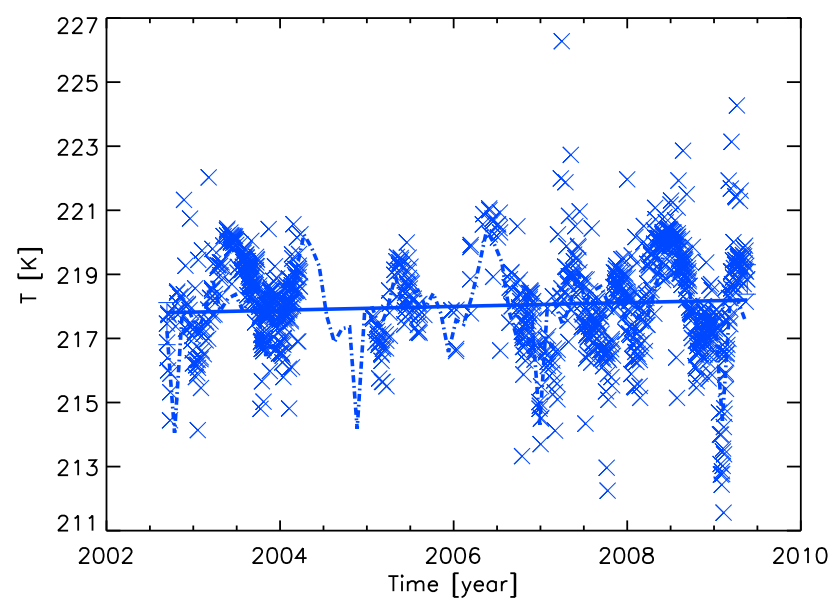

Fig. 4. Linear trends from MIPAS daily mean temperatures at $25 \mathrm{~km}$ altitude, $30^{\circ} \mathrm{S}-30^{\circ} \mathrm{N}$, using the continuous empirical periodical correction, as described in Sect. 3.3. Both the linear part of the regression function (solid line) and the complete regression function (dash dot) are shown.

$$
\begin{aligned}
& \hat{y}(x ; a, b, c, d)=a+b x \\
& +(a+b x) c \sin \frac{2 \pi x}{l}+ \\
& (a+b x) d \cos \frac{2 \pi x}{l} \\
& =a+b x \\
& +a c \sin \frac{2 \pi x}{l}+b x c \sin \frac{2 \pi x}{l}+ \\
& a d \cos \frac{2 \pi x}{l}+b x d \cos \frac{2 \pi x}{l}
\end{aligned}
$$

This is typically the case with concentrations of atmospheric constituents: When the average abundance is larger, also the diurnal or annual cycle is expected to have a larger amplitude. Minimization of the related cost function leads to a nonlinear system of equations, because $x$ appears both in the argument of the trigonometric function and in its multiplier. Since for such problems there exists no straight-forward closed-form solution, this system of nonlinear equations is suggested to be linearized and iteratively solved for $a_{i+1}, b_{i+1}, c_{i+1}$, and $d_{i+1}$, where $i$ is the iteration index. The dependence of the amplitudes of the periodic components on the actual state are calculated from the results of the preceding iteration step:

$$
\begin{aligned}
0= & -2 \boldsymbol{e}^{T} \mathbf{S}_{y}^{-1}\left(\boldsymbol{y}(\boldsymbol{x})-a_{i+1} \boldsymbol{e}-b_{i+1} \boldsymbol{x}-\right. \\
& c_{i+1} \operatorname{diag}\left(\left(a_{i} \boldsymbol{e}+b_{i} \boldsymbol{x}\right) \boldsymbol{v}_{\mathrm{sin}}^{T}\right)- \\
& \left.d_{i+1} \operatorname{diag}\left(\left(a_{i} \boldsymbol{e}+b_{i} \boldsymbol{x}\right) \boldsymbol{v}_{\mathrm{cos}}^{T}\right)\right)
\end{aligned}
$$




$$
\begin{aligned}
& 0=-2 \boldsymbol{x}^{T} \mathbf{S}_{y}^{-1}\left(\boldsymbol{y}(\boldsymbol{x})-a_{i+1} \boldsymbol{e}-b_{i+1} \boldsymbol{x}-\right. \\
& c_{i+1} \operatorname{diag}\left(\left(a_{i} \boldsymbol{e}+b_{i} \boldsymbol{x}\right) \boldsymbol{v}_{\sin }^{T}\right)- \\
& \left.d_{i+1} \operatorname{diag}\left(\left(a_{i} \boldsymbol{e}+b_{i} \boldsymbol{x}\right) \boldsymbol{v}_{\mathrm{cos}}^{T}\right)\right) \\
& 0=-2\left(\operatorname{diag}\left(\left(a_{i} \boldsymbol{e}+b_{i} \boldsymbol{x}\right) \boldsymbol{v}_{\sin }^{T}\right)\right)^{T} \mathbf{S}_{y}^{-1} \times \\
& \left(\boldsymbol{y}(\boldsymbol{x})-a_{i+1} \boldsymbol{e}-b_{i+1} \boldsymbol{x}-\right. \\
& c_{i+1} \operatorname{diag}\left(\left(a_{i} \boldsymbol{e}+b_{i} \boldsymbol{x}\right) \boldsymbol{v}_{\sin }^{T}\right)- \\
& \left.d_{i+1} \operatorname{diag}\left(\left(a_{i} \boldsymbol{e}+b_{i} \boldsymbol{x}\right) \boldsymbol{v}_{\mathrm{cos}}^{T}\right)\right) \\
& 0=-2\left(\operatorname{diag}\left(\left(a_{i} \boldsymbol{e}+b_{i} \boldsymbol{x}\right) \boldsymbol{v}_{\mathrm{cos}}^{T}\right)\right)^{T} \mathbf{S}_{y}^{-1} \times \\
& \left(\boldsymbol{y}(\boldsymbol{x})-a_{i+1} \boldsymbol{e}-b_{i+1} \boldsymbol{x}-\right. \\
& c_{i+1} \operatorname{diag}\left(\left(a_{i} \boldsymbol{e}+b_{i} \boldsymbol{x}\right) \boldsymbol{v}_{\sin }^{T}\right)- \\
& \left.d_{i+1} \operatorname{diag}\left(\left(a_{i} \boldsymbol{e}+b_{i} \boldsymbol{x}\right) \boldsymbol{v}_{\mathrm{cos}}^{T}\right)\right) \text {, }
\end{aligned}
$$

Again the equation involving $\partial \chi^{2} / \partial a=0$ is replaced by $c_{1}+\ldots+c_{J}=0$. The iteration can be initialized with

$a_{0}=\sum_{n=1}^{N} \frac{y_{n}}{N}$

and

$b_{0}=0$

and is supposed to converge for cases where the trend is sufficiently small and/or the amplitude of the superimposed periodic function is sufficiently small compared to the $y_{n}$ values. In both cases, the time-dependence of the amplitude is only a small perturbation of a function dominated by its linear terms. Similar considerations apply to periodic corrections as suggested in Eqs. (27) and (31).

\section{Case Study 5:}

The approach involving state-dependent amplitudes of periodic corrections is applicable particularly to trace gas abundances rather than temperature. We use MIPAS southern polar $\left(60^{\circ}-90^{\circ} \mathrm{S}\right)$ monthly $\mathrm{CFC}-11$ mean mixing ratios at $20 \mathrm{~km}$ altitude. Its negative trend is a consequence of the

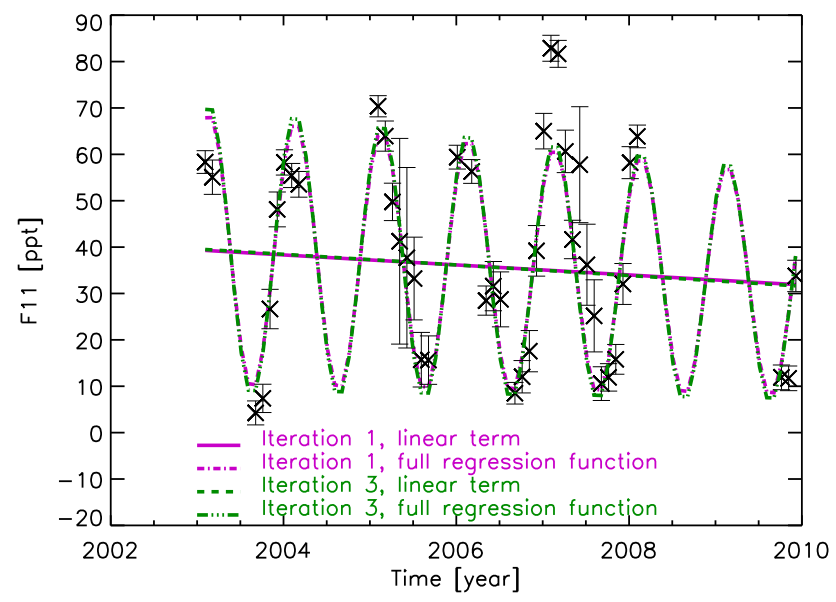

Fig. 5. Linear trends from MIPAS monthly mean CFC-11 at $20 \mathrm{~km}$ altitude, $60^{\circ} \mathrm{S}-90^{\circ} \mathrm{S}$, using periodical correction of state-dependent amplitude, as described in Sect. 3.4. The fit converges after the third iteration.

Montreal Protocol, and the annual variation is driven by atmospheric circulation and, particularly, subsidence of CFCdepleted air in the polar winter stratosphere. Since the efficiency of loss reactions leading to CFC-depletion is proprotional to its abundance, application of a correction function of an abundance-dependent amplitude is appropriate. Also in this case the change in the MIPAS measurement mode in 2004 makes the dataset inhomogeneous. In the related covariance matrix we consider an unknown bias between the data subsets of $\pm 2 \mathrm{ppt}$. Due to the moderate trend the iteration converges rapidly. The regression function after the first iteration is

$$
\begin{aligned}
& {[\mathrm{CFC}-11] / \mathrm{ppt}=} \\
& 42.62-1.08 t+ \\
& (42.62-1.08 t)(0.575 \sin (2 \pi t)+0.491 \cos (2 \pi t)),
\end{aligned}
$$

where $t$ is the time since 2000 in years. After the third iteration the final regression function is reached (Fig. 5):

$$
\begin{aligned}
& {[\mathrm{CFC}-11] / \mathrm{ppt}=} \\
& 43.04-1.15 t+ \\
& (43.04-1.15 t)(0.599 \sin (2 \pi t)+0.522 \cos (2 \pi t))
\end{aligned}
$$

The uncertainty of the trend is $\pm 0.38 \mathrm{ppt} / \mathrm{yr}$.

\section{Conclusions}

In case of irregular temporal and spatial sampling and/or multiple measurement systems, intersite and/or intersystem error correlations have to be considered for trend estimation. To disregard the correlations not only renders the significance analysis meaningless, but leads to wrong estimates of the trend itself. Intersite correlations as well as correlation lengths in the time domain can be estimated from satellite data, where, however, the limited spatial resolution of 
remote measurements can add some difficulty. The regression model can easily be adapted for periodic corrections of known period length but unknown phase, shape and amplitude. This scheme solves the problem that usual approaches to infer periodic corrections rely on the time series being stationary, which is inherently not true in the case of trend estimation. State-dependent amplitudes of superimposed periodic corrections can be appropriate when trends of trace gas abundances are evaluated and result in a non-linear optimization problem which is solved iteratively.

Acknowledgements. ESA has provided MIPAS level-1B data.

Edited by: W. Lahoz

\section{References}

Aitken, A. C.: On Least squares and linear combinations of observations, P. Roy. Soc. Edinb., 55, 42-48, 1935.

Baldwin, M. P., Gray, L. J., Dunkerton, T. J., Hamilton, K., Haynes, P. H., Randel, W. J., Holton, J. R., Alexander, M. J., Hirota, I., Horinouchi, T., Jones, D. B. A., Kinnersley, J. S., Marquardt, C., Sato, K., and Takahashi, M.: The quasi-biennial oscillation, Rev. Geophys., 39, 179-229, 2001.

Cantrell, C. A.: Technical Note: Review of methods for linear leastsquares fitting of data and application to atmospheric chemistry problems, Atmos. Chem. Phys., 8, 5477-5487, doi:10.5194/acp8-5477-2008, 2008.

Cox, M., Harris, P., Hilton, M., and Woods, P.: Method for evaluating trends in ozone concentration data and its application to data from the UK Rural Ozone Monitoring Network, NPL Report CMSC 15/02, 2002.

Efron, B.: The 1977 Rietz lecture: Bootstrap methods: Another look at the jackknife, Ann. Stat., 7, 1-26, 1979.

Engel, A., Möbius, T., Bönisch, H., Schmidt, U., Heinz, R., Levin, I., Atlas, E., Aoki, S., Nakazawa, T., Sugawara, S., Moore, F., Hurst, D., Elkins, J., Schauffler, S., Andrews, A., and Boering, K.: Age of stratospheric air unchanged within uncertainties over the past 30 years, Nat. Geosci., 2, 28-31, 2009.

Fischer, H., Birk, M., Blom, C., Carli, B., Carlotti, M., von Clarmann, T., Delbouille, L., Dudhia, A., Ehhalt, D., Endemann, M., Flaud, J. M., Gessner, R., Kleinert, A., Koopman, R., Langen, J., López-Puertas, M., Mosner, P., Nett, H., Oelhaf, H., Perron, G., Remedios, J., Ridolfi, M., Stiller, G., and Zander, R.: MIPAS: an instrument for atmospheric and climate research, Atmos. Chem. Phys., 8, 2151-2188, doi:10.5194/acp-8-2151-2008, 2008.

Gardiner, T., Forbes, A., de Mazière, M., Vigouroux, C., Mahieu, E., Demoulin, P., Velazco, V., Notholt, J., Blumenstock, T., Hase, F., Kramer, I., Sussmann, R., Stremme, W., Mellqvist, J., Strandberg, A., Ellingsen, K., and Gauss, M.: Trend analysis of greenhouse gases over Europe measured by a network of ground-based remote FTIR instruments, Atmos. Chem. Phys., 8, 6719-6727, doi:10.5194/acp-8-6719-2008, 2008.

Guharay, A., Nath, D., Pant, P., Pande, B., Russell III, J. M., and Pandey, K.: Observation of semiannual and annual oscillation in equatorial middle atmospheric long term temperature pattern, Ann. Geophys., 27, 4273-4280, doi:10.5194/angeo-274273-2009, 2009.
IPCC: Climate Change 2007: The Physical Science Basis, Contribution of Working Group I to the Fourth Assessment Report of the Intergovernmental Panel on Climate Change, edited by: Solomon, S., Qin, D., Manning, M., Chen, Z., Marquis, M., Averyt, K. B., Tignor M., and Miller, H. L., Cambridge University Press, Cambridge, UK and New York, NY, USA, 996 pp., 2007.

Jones, P. D., Osborn, T. J., and Briffa, K. R.: Estimating sampling errors in large-scale temperature averages, J. Climate, 10, 25482568, 1997.

Milz, M., von Clarmann, T., Fischer, H., Glatthor, N., Grabowski, U., Höpfner, M., Kellmann, S., Kiefer, M., Linden, A., Mengistu Tsidu, G., Steck, T., Stiller, G. P., Funke, B., López-Puertas, M., and Koukouli, M. E.: Water vapor distributions measured with the Michelson Interferometer for Passive Atmospheric Sounding on board Envisat (MIPAS/Envisat), J. Geophys. Res., 110, D24307, doi:10.1029/2005JD005973, 2005.

Milz, M., Clarmann, T. v., Bernath, P., Boone, C., Buehler, S. A., Chauhan, S., Deuber, B., Feist, D. G., Funke, B., Glatthor, N., Grabowski, U., Griesfeller, A., Haefele, A., Höpfner, M., Kämpfer, N., Kellmann, S., Linden, A., Müller, S., Nakajima, H., Oelhaf, H., Remsberg, E., Rohs, S., Russell III, J. M., Schiller, C., Stiller, G. P., Sugita, T., Tanaka, T., Vömel, H., Walker, K., Wetzel, G., Yokota, T., Yushkov, V., and Zhang, G.: Validation of water vapour profiles (version 13) retrieved by the IMK/IAA scientific retrieval processor based on full resolution spectra measured by MIPAS on board Envisat, Atmos. Meas. Tech., 2, 379399, doi:10.5194/amt-2-379-2009, 2009.

Muhlbauer, A., Spichtinger, P., and Lohmann, U.: Application and comparison of robust linear regression methods for trend estimation, J. Appl. Meteorol. Climl., 48, 1961-1970, doi:10.1175/2009JAMC1851.1, 2009.

Randel, W. J., Wu, F., Oltmans, S. J., Rosenlof, K., and Nedoluha, G. E.: Interannual changes of stratospheric water vapor and correlations with tropical tropopause temperatures, J. Atmos. Sci., 61, 2133-2148, 2004.

Reed, R. J.: The quasi-biennial oscillation of the atmosphere between 30 and $50 \mathrm{~km}$ over Ascension Island, J. Atmos. Sci., 22, 331-333, 1965.

Rosenlof, K. H. and Reid, G. C.: Trends in the temperature and water vapor content of the tropical lower stratosphere: Sea surface connection, J. Geophys. Res., 113, D06107, doi:10.1029/2007/JD009109, 2008.

Vigouroux, C., De Mazière, M., Demoulin, P., Servais, C., Hase, F., Blumenstock, T., Kramer, I., Schneider, M., Mellqvist, J., Strandberg, A., Velazco, V., Notholt, J., Sussmann, R., Stremme, W., Rockmann, A., Gardiner, T., Coleman, M., and Woods, P.: Evaluation of tropospheric and stratospheric ozone trends over Western Europe from ground-based FTIR network observations, Atmos. Chem. Phys., 8, 6865-6886, doi:10.5194/acp-8-68652008, 2008.

von Clarmann, T., Glatthor, N., Grabowski, U., Höpfner, M., Kellmann, S., Kiefer, M., Linden, A., Mengistu Tsidu, G., Milz, M., Steck, T., Stiller, G. P., Wang, D. Y., Fischer, H., Funke, B., GilLópez, S., and López-Puertas, M.: Retrieval of temperature and tangent altitude pointing from limb emission spectra recorded from space by the Michelson Interferometer for Passive Atmospheric Sounding (MIPAS), J. Geophys. Res., 108(D23), 4736, doi:10.1029/2003JD003602, 2003. 
von Clarmann, T.: Validation of remotely sensed profiles of atmospheric state variables: strategies and terminology, Atmos. Chem. Phys., 6, 4311-4320, doi:10.5194/acp-6-4311-2006, 2006.

von Clarmann, T., Höpfner, M., Kellmann, S., Linden, A., Chauhan, S., Funke, B., Grabowski, U., Glatthor, N., Kiefer, M., Schieferdecker, T., Stiller, G. P., and Versick, S.: Retrieval of temperature, $\mathrm{H}_{2} \mathrm{O}, \mathrm{O}_{3}, \mathrm{HNO}_{3}, \mathrm{CH}_{4}, \mathrm{~N}_{2} \mathrm{O}, \mathrm{ClONO}_{2}$ and $\mathrm{ClO}$ from MIPAS reduced resolution nominal mode limb emission measurements, Atmos. Meas. Tech., 2, 159-175, doi:10.5194/amt-2-1592009, 2009.
Weatherhead, E. C., Reinsel, G. C., Tiao, G. C., Meng, X.-L., Choi, D., Cheang, W.-K., Keller, T., DeLuisi, J., Wuebbles, D. J., Kerr, J. B., Miller, A. J., Oltmans, S. J., and Frederick, F. E.: Factors affecting the detection of trends: Statistical considerations and applications to environmental data, J. Geophys. Res., 103, 17149-17161, 1998. 